\title{
Investigation of Haemostatic Status in the Elderly Male and Female Using Some Parameters; PT, APTT and PC Count (A Case Study in Owerri Metropolis)
}

\author{
Okoroiwu Ijeoma Leticia ${ }^{1}$, Obeagu Emmanuel Ifeanyi ${ }^{2}$ and \\ Elemchukwu Queen ${ }^{3}$, Ochei Kingsley Chinedum ${ }^{4}$ \\ 1. Lecturer (P.hD), Department of Medical Laboratory Science,Faculty of Health Science,Imo State \\ University, Owerri, Nigeria. \\ 2. Diagnostic Laboratory Unit, University Health services Department, Michael Okpara University of \\ Agriculture,Umudike, Nigeria. \\ 3 Rivers State College of Health Science and Technology, Port Harcourt \\ 4.Department of Medical Laboratory Science,Ambrose Alli University,Ekpoma Edo State, Nigeria
}

\begin{abstract}
The effect of age on prothrombin time (PT) activated partial thromboplastin time (APTT) and platelet count (PC) was investigated. Forty male and female above 60 years where sampled. Their prothrombin time, activated partial thromboplastin time, and platelet counts were determined. The forty candidates were made up of twenty male and twenty female. In addition, twenty control subjects made up of ten male and ten female below the age of 25 years were also investigated. Both the elderly male and female were graded from 60 65, 65-70 and 70 and above. At the time of this research, the people investigated were ambulant and free from carcinoma, thrombosis, hypertension, diabetes mellitus and liver disease. The mean prothrombin time for the 60-65 groups was $15.5 \pm 1.3$ for elderly male and $15.0 \pm 0.6$ for elderly female. The mean prothrombin time for the 65-70 groups was $16.5 \pm 1.7$ and $15.5 \pm 0.9$ for elderly male and female respectively. For 70 and above group, it was $18.0 \pm 1.3$ and $16.5 \pm 1.9$ for elderly male and female respectively. The means of all the three groups together

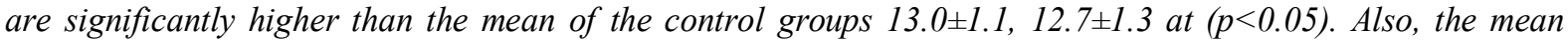
APTT for elderly male at 60-65, 65-70 and 70 years and above were $35.5 \pm 0.3,36 \pm 1.4$ and $37.0 \pm 1.5$ respectively. The mean APTT for elderly male at $60-65,65-70$ and 70 years and above were $33.5 \pm 0.4,35.0 \pm 1.5$ and $36.5 \pm 1.2$ respectively. When the means were compared with the means of male (34.0 \pm 0.7$)$ and female control (32.0 \pm 0.6$)$, it showed a significant increase in APTT in both elderly male and female at $(p<0.05)$. But there is a marked decrease in levels of the platelet count. This is shown by the mean platelet counts in elderly male of about $205 \pm 5 \times 10^{9} / \mathrm{L}, 201 \pm 8 \times 10^{9} / \mathrm{L}$ and $185 \pm 7 \times 10^{9} / \mathrm{L}$ at $60-65,65-70$ and 70 years and above respectively as against the mean platelet counts of $254 \pm 11 \times 10^{9} \mathrm{~L}$ recorded in male controls. The mean counts of $186 \pm 9 \times 10^{9} / \mathrm{L}, 182 \pm 10^{9} / \mathrm{L}$ and $155 \pm 10^{9} / \mathrm{L}$ recorded in elderly female at $60-65,65-70$, and 70 years and above respectively as compared to the mean count of $260 \pm 12 \times 10^{9} / \mathrm{L}$ recorded in female control. The decrease is more pronounced in elderly female than in male.
\end{abstract}

Keywords: Prothrombin time, APTT, platelet counts and age.

\section{Introduction}

Haemostasis as a word is taken from the Greek word, Haem meaning blood and stasis meaning standing. It is a process which causes bleeding to stop thereby stopping blood from escaping through damaged blood vessel. It is the first stage of wound healing, most of the time this includes blood changing from a liquid to solid state. Intact blood vessels are central to moderating blood's tendency to clot. The endothelial cells of intact vessels prevent blood clotting with a heparin like molecule and thrombomodulin which prevents platelet aggregation with nitric oxide and prostacyclin (Marieb et al., 2010). When endothelia injury occurs, the endothelial cells stops secretion of coagulation and aggregation inhibitors and instead secrete Von Willbrand factors which initiate the maintenance of haemostasis after injury.

Haemostasis is very important because it arrests bleeding (clot formation) when blood vessels are damaged which if the bleeding is not arrested (sudden and severe loss of blood) can lead to shock and death.

Haemostasis is the instinctive response for the body to stop bleeding and loss of blood. During haemostasis three steps occur in a rapid sequence. Vascular spasm is the first response as the blood vessels constricts to allow less blood to be lost. In the second step, platelets plug formation, platelet stick together to form a temporary seal to cover the break in the vessel wall. The third and last step is called coagulation or blood clotting. Coagulation reinforces the platelets plug with fibrin threads that act as a "molecular glue" (Marieb et al., 2010). Platelets are large factors in the haemostatic process. They allow for the creation of the "Platelet plug" that forms almost directly after a blood vessel has been ruptured. Within seconds of a blood vessel's 
Investigation of Haemostatic Status in the Elderly Male and Female Using Some Parameters; Pt...

epithelial wall being disrupted, platelets begin to adhere to the sub-endothelium surface. It takes approximately sixty seconds until the first fibrin strands begin to intersperse among the wound. After severe minutes the platelet plug is completely formed. (Boon, 1993). Homeostasis is maintained in the body via three mechanisms. (1). Vascular spasm is the blood vessels first response to injury. The damaged vessels will constrict (vasoconstriction) which reduces the amount of blood flow through the area and limit the amount of blood loss. This response is triggered by factor such as a direct injury to vascular smooth muscle, chemicals released by endothelial cells and platelet, and reflexes initiated by local pain receptors. The spasm response becomes more effective as the amount of damage is increased.

(2). Platelet plug formation, when the vessel wall is damaged, the endothelial structures, including basement membrane, collagen and microfibrils are exposed. Surface bound vonwillebrand factor binds to glycoprotein $1 \mathrm{~b}$ on circulating. Platelets resulting in an initial monolayer of adhering platelets. Being the second step in the sequence they stick together (aggregation) to form a plug that temporarily seals the break in the vessel wall. As platelets adhere to the collagen fibers of a wound they become spiked and much stickier. They then release chemical messenger such as adenosine disphophate (ADP).

These chemicals are released to cause more platelets to stick to the area and release their contents and enhance vascular spasm. Platelets alone are responsible for stopping the bleeding of unnoticed wears and tears of our skin on a daily basis (Clemetson et al., 2012) platelet plug formation is activated by glycoprotein called the von willebrand factor which are found in the blood plasma. When platelets in the blood are activated they then become very sticky so allowing them to stick to other platelets and adhere to the injured area (Lassila et al., 2012).

(3). Blood coagulation- Clots form upon the conversion of fibrinogen to fibrin, and its addition to the platelet plug (secondary hemostasis). Coagulation which is the third and final step in its rapid response reinforces the platelet plug. Coagulation uses fibrin threads that act as a glue for the sticky platelets. As the fibrin mesh begins to form the blood is also transformed from a liquid to a gel like substance through involvement of clotting factors and pro-coagulates.

\section{HAEMOSTATIC FACTORS AND AGING}

With advancing age, many individuals who are otherwise normal show laboratory evidence of heightened coagulation enzyme activity.

The lecture of D-Maris on haemostasis and aging held on (March 19 2008) says, physiological aging is associated with increased plasma levels of many protein of blood coagulation with fibrinolysis impairment. This may be of great concern in view of the known association between vascular and thromboembolic diseases and aging. Prothrombotic clotting factor; the plasma concentration of several clotting factors namely fibrinogen factor vii, factor viii, Von willebrand factor (VWF) factor ix, factor xii increase with progressing age in healthy individuals (Maris et al., 2008). A study by (Meade et al., 1977) in a population study of subjects aged 5364 years had shown significantly higher level of fibrinogen $(300 \mathrm{mg} / \mathrm{dl})$ than those found in younger subjects aged $20(250 \mathrm{mg} / \mathrm{dl})$. As $10 \mathrm{mg} / \mathrm{dl}$ for each decade can be expected in healthy subjects. Fibrinogen moreover is a molecule that plays a role in acute phase inflammation and fibrinogen level increases in reference to interleukin in 6 and both are strongly connected with aging (Barleisen et al., 1985). Factor vii plasma levels progressively increase with age from a mean of 95 units/dl in subjects of 20 years old to over 110 units/dl in subjects over 50 years old. Thrombotic disorders have shown to be more frequent in subjects with higher plasma levels of factor vii (Ershler, 1993) acting as a cofactor in the activation of factor X promoted by factor IXa, progressing increase with age reaching a mean of over 200 units/dl in the healthy subjects over sixty of age, the level of factor ix and factor X activation peptide also increase with advancing age (Bauer et al., 1990). The mean physiological inhibitors of blood coagulation are natural anticoagulants produced by the liver and circulating in the plasma, anti thrombin iii heparin co-factor ii. The protein $\mathrm{C}$, protein $\mathrm{S}$ system and tissue factor pathway inhibitors (T.F.P.I). The increase activation of the related coagulation is not the tissue factors pathway, which increase with increase in age, the behavior pattern of TFPI is gender dependent, in women statistically significant increase in plasma concentration of TFPI with age have been observed paralleling the rise in factor Vii. No significant age-related change in TFPI has been found in men (Ariensm et al., 1995).

\section{JUSTIFICATION}

Haemostasis is a process which stops bleeding. Haemostasis in the elderly is a research work to determine the haemostatic status in the elderly subjects specifically their platelet status, prothrombin status and activated partial thromboplastin time status. Many research works has been done on haemostasis but not much on geriatrics especially in this part of the world especially owerri considering the social, economic and demorgraphyical differences in the study areas. Hence the need for this work.

\section{AIMS AND OBJECTIVES}


1) To investigate the haemostatic status in the elderly individuals of 60 years and above using some parameters like prothrombin time (PT), activated partial thromboplastin time (APTT) and platelet count.

2) To compare the result obtained with the normal control values of men and women below 25 years $(20-25$ years).

\section{STUDY AREA}

\section{Materials And Methods}

This project work was carried out at the Federal Medical Center (FMC) Owerri, Imo State between the months of November to December. Owerri is located in the tropic Rain forest of South East Nigeria; it lies on the $5^{\circ} 27-50^{\circ}, 31^{\circ} \mathrm{N}$ and longitude $6058-70^{\circ} \mathrm{SE}$. It has a population of about $(75,21535278$ male and 39937 female) and it's comsmopolitan being home to many non indigenes apart from the ethnic Igbo which is a major ethnic group area. The inhabitants are mainly Christian, few Muslims and pagans scattered within the area. The climate o the area is tropical with its mean daily temperature of $29 \pm 50^{\circ \mathrm{cc}}$ for the year.

\section{STUDY POPULATION}

A total of 60 subjects were used for this study, which consist of both test and control groups. The subjects were drawn from apparently healthy elderly people whose ages ranges from 60 years and above and younger controls whose ages ranges from 20 to 25 years.

\section{SPECIMEN COLLECTION AND METHOD}

$5 \mathrm{mls}$ of blood were collected from each elder and this was withdrawn without stasis for the investigation of prothrombin time, 9 parts of blood and 1 part of trisodium citrate was used $(0.5 \mathrm{ml}$ of anticoagulant for a $4.5 \mathrm{ml}$ specimen) this was mixed immediately after extraction and centrifuged for $1500 \mathrm{~g}$ to obtain a plasma poor in platelet.

Tests were performed not later two hours after collection. The remaining blood in the syringes was added in Ethylene diamine tetra acetic tube (EDTA) for platelet count.

\section{PROTHROMBIN TIME (PT)}

(Quick one stage method adopted Dacie and Lewis, 2012)

\section{PROCEDURE}

The PT reagent was reconstituted by adding $5 \mathrm{mls}$ of its diluents mixed by inversion and allowed to stand at room temperature for 30 mines. The reconstituted PT reagent was pre-warmed at $37^{0 \mathrm{c}}$ for 10 mines. $50 \mu \mathrm{l}$ of sample was added into the test curette and incubated for 2 mines at $37^{\circ \text { c }}$, then $100 \mu l$ of the incubated PT reagent was rapidly added and the time of clotting in seconds was recorded.

\section{ACTIVATED PARTIAL THROMBOPLASTIN TIME}

(APTT) adopted from Dacie and Lewis 2012 modified Kaolin method.

\section{PROCEDURE}

The APTT reagent was reconstituted with $4 \mathrm{mls}$ of distilled water, mixed by inversion and allowed to stand at room temperature for 30 mins. $50 \mathrm{ul}$ of the sample was added into a test corvette, then $50 \mathrm{ul}$ of the sample, the mixture was incubated for $30 \mathrm{mins}$ at $37 \mathrm{oc}$. $50 \mathrm{ul}$ of calcium chloride was rapidly added and the time of clotting in seconds was recorded.

\section{PLATELET}

Direct current method

By automation using sysmex automated heamatology analyser KY2IN model manufactured by Sysmex Corporation Kobe Japan.

Procedure:

The sample in EDTA bottle was placed in the spiral mixer and allowed to mix very well, whole blood mode was activated in the LCD screen, the sample no (code) was inputted via the key board and then the enter key was entered. Then the sample was mixed very well again, the cap was removed and inserted into the probe, on that condition, the start switch was pressed. The LCD screen displayed analyzing, the unit sample was removed sand recapped the unit executes automatic analysis and displays the result on the LCD screen.

\section{STATISTICAL ANALYSIS}

All data was statistically evaluated by using SPSS version 20.0 (statistical package for social sciences). Values were expressed as mean \pm standard deviation. The results were analyzed for statistical significance using the independent student T-test P-value $<0.05$ were considered statistically significant.

\section{Results}

Table 4.1: The table below compares the mean prothrombin time and standard deviation of the different age brackets and sex including the appropriate controls.

\begin{tabular}{|l|l|l|l|}
\hline Age Range & Number & Mean Time(seconds) & Standard Deviation (s) \\
\hline $60-65$ & 4 male & 15.5 & \pm 1.3 \\
\hline $60-70$ & 7 female & 15.0 & \pm 0.6 \\
\hline
\end{tabular}


Investigation of Haemostatic Status in the Elderly Male and Female Using Some Parameters; Pt...

\begin{tabular}{|l|l|l|l|}
\hline & 7 female & 15.5 & \pm 0.9 \\
\hline 70 and above & 9 male & 18.0 & \pm 1.3 \\
\hline & 6 female & 16.5 & \pm 1.9 \\
\hline Control & 10 male & 13.0 & \pm 1.1 \\
\hline & 10 female & 12.7 & \pm 1.3 \\
\hline
\end{tabular}

Table 4.2: The table below shows the mean activated partial thromboplastin time and standard deviation of the different age brackets and sex, including the controls.

\begin{tabular}{|l|l|l|l|}
\hline Age Range & Number & Mean Time (seconds) & Standard Deviation (s) \\
\hline $60-65$ & 4 male & 35.5 & \pm 0.3 \\
\hline & 7 female & 33.5 & \pm 0.4 \\
\hline $65-70$ & 7 male & 36.5 & \pm 1.4 \\
\hline & 7 female & 35.0 & \pm 1.5 \\
\hline 70 and above & 9 male & 37.0 & \pm 1.5 \\
\hline & 6 female & 36.5 & \pm 1.2 \\
\hline Controls & 10 male & 34.0 & \pm 0.7 \\
\hline & 10 female & 32.0 & \pm 0.6 \\
\hline
\end{tabular}

Table 4.3: The table below compares the mean count of platelet and standard deviation between the elderly male and female with their appropriate controls

\begin{tabular}{|l|l|l|l|}
\hline Age Range & Number & Mean count X $10^{9} / \mathrm{L}$ & Standard Deviation (s) \\
\hline $60-65$ & 4 male & 205 & \pm 5.0 \\
\hline & 7 female & 186 & \pm 9.0 \\
\hline $65-70$ & 7 male & 201 & \pm 8.0 \\
\hline & 7 female & 162 & \pm 10.0 \\
\hline 70 and above & 9 male & 185 & \pm 7.0 \\
\hline & 6 female & 155 & \pm 9.0 \\
\hline Control & 10 male & 254 & \pm 11.0 \\
\hline & 10 female & 260 & \pm 12.0 \\
\hline
\end{tabular}

\section{Discussion}

The effect of age on prothrombin time PT activated partial thromboplastin time (APTT) and platelet count was investigated in the elderly male and female. From the tables, the mean prothrombin time for elderly male at 60-65 years, $65-70$ years and 70 years and above were $15.5 \pm 1.3,16.5 \pm 1.7$ and $18.0 \pm 1.3$ respectively when the mean were compared with the mean of the male control (13.0 11.1$)$, it showed a significant increase $(\mathrm{p}<0.05)$ in prothrombin time. Also, the mean prothrombin time for elderly women at $60-65,65-70$ and 70 years and above were $15.0 \pm 0.6,15.5 \pm 0.9$ and $16.5 \pm 1.9$ respectively. When the means were compared with the mean of the female control $(12.7 \pm 1.3)$, it showed a significant increase $(\mathrm{p}<0.05)$ in prothrombin time. Therefore, prothrombin time increases with age (Hamilton et al., 1974). The significant change suggests the decrease of the extrinsic pathway ( $\mathrm{F}$ vii) but mild factor $\mathrm{X}, \mathrm{V}$, and II as age process progresses .

Also from the table, the mean activated partial thromboplastin time for elderly male at 60-65 years, 6570 years, and 70 years and above were $35.3 \pm 0.3,36.5 \pm 1.4$ and $37.0 \pm 1.5$ respectively. When the mean were compared with the mean of the male control $(34.0 \pm 0.7)$, it showed a significant increase at $(\mathrm{p}<0.05)$ in activated partial thromboplastin time.

The mean APTT for elderly women from the tables at 60-65 years, 65-70 years and 70 years and above were $33.5 \pm 0.4,35.0 \pm 1.5$ and $36.5 \pm 1.2$ respectively when the means were compared with the mean of female control $(32.0 \pm 0.6)$, it showed a significant increase at $(\mathrm{p}<0.05)$ in activated partial thromboplastin time. The significant changes in activated partial thromboplastin time is attributed to the decrease of one or more of the intrinsic pathway clotting factors (prekallikrein, high molecular weight kininogen, factor XII, XI, IX, and VIII. In all the increase of both APTT and PT suggests decrease of the common pathway coagulation factor (factor X, $\mathrm{V}$, and II) or a qualitative or quantitative fibrinogen defect as age process progresses.

Also, the occurrence of menopause in elderly women is accompanied by a significant increase in antithrombin III plasma level coagulation system (Sapripanti and Carpi, 1999). Protein S. and protein C also rise with age in both sexes.

In contrast to the overall effect of aging to increased prothrombin time and activated partial thromboplastin time, is has been shown statistical that there is a marked decrease in the number of platelet counts as aging progresses. This is shown by the mean count of $205 \pm 5 \times 10^{9} / \mathrm{L}, 201 \pm 8 \times 10^{9} / \mathrm{L}$ and $185 \pm 7 \times 10^{9} / \mathrm{L}$ recorded in elderly men at 60-65, 65-70 years and above respectively as compared to the mean count of $254 \pm 11 \times 10^{9} / \mathrm{L}$ recorded in male controls. The marked decrease in levels of platelet counts can also be shown by the mean counts of $186 \pm 9 \times 10^{9} / \mathrm{L}, 162 \pm 10 \times 10^{9} / \mathrm{L}$ and $155 \pm 9 \times 10^{9} / \mathrm{L}$ recorded in elderly women of $60-65,65-70$ and 70 years and above respectively as compared to the mean count of $260 \pm 12.0 \times 109 / \mathrm{L}$ recorded in female controls. The decrease in platelet counts is more pronounced in elderly women than in elderly men due to a 
decreased sensitivity to the specific inhibitor rotenone which affects the level of platelet counts (Merlo-Pich et al., 1996).

The effect of aging on platelet may be related to reduce hematopoietic stem cell reverse in aging individuals (Segal et al., 2006). The decrease in platelet during aging is also attributed to age dependent decline in platelet number which effect epigenetic changes in the megakaryocyte genome. (Buckley et al., 2000). From this analysis, it is convenient to state that in the elderly, there is an increase in prothrombin time (PT) and activated partial thromboplastin (APTT) while the platelet counts show a significant decrease when compared with their younger controls.

\section{Conclusion}

From the investigation carried out on the effect of age on haemostatic status in the elderly, it is convenient to state that in the elderly, there is an increase in prothrombin time (PT) and activated partial thromboplastin time (APTT) while the platelet counts show a significant decrease when compared with the younger controls.

Finally, from the finding of the work the results suggest that any study of the coagulation parameters must consider age related changes and provide standard against which such results from elderly people can be evaluated.

\section{References}

[1]. Ariensm R., Coppola, R., Potenza, I., Mannucci, P. (1995): The Increase with age of the components of the tissue Factor coagulation pathway is dependent. Blood Coagulation fibrinolysis . 6: 433-37

[2]. Bauer, K.A., Kass, B.L., Cate, H.C., Hawiger, J.J. (1990): Rosenberg RD: factor ix is activated in vivo by the tissue factor mechanism. Blood. 76: 731-36.

[3]. Balleisen, L., Bailey, J., Epping, P.H., Schulte, H., Loo, J. (1985): Epidemiological study on factor vii, factor viii and fibrinogen in industrial population. Baseline data on the relation to age, gender, body-weight, smoking, alcohol, pill using and menopause. Throm. Haemost. 45: 475-79.

[4]. Boon, E.D., (1993): “An overview of hemostasis”. Toxicologic Pathology. 21 (2): 170-79.

[5]. Bukley, M.F., James, J.W., Brown, D.E., Whyte, G.S., Dean, M.E., Chesterman, C.N., Donald, J.A. (2000): A novel approach to the assessment of variations in the human platelet count. Thromb Haemost. 83(3): 480-4

[6]. Clemetson, K.J. (2012): "Platelets and primary haemostasis". Thrombosis Research. 129 (3): 220 - 224

[7]. Ershler, W.B. (1983): Intetleukin-6, a cytokine for gerontologist.J.A.M. Geriatr Soc. 41: 179 - 181 Lassila, R. (2012): New Insights into von willebrand Disease and Platelet function. Seminars in the thrombosis research. 38(1): 55-63.

[8]. Marieb, E., Haelin, K. (2010): Human Anatomy and physiology $8^{\text {th }}$ ed.). San Francisco Benjamin Cummings. $649-50$.

[9]. Maris, D., Coppola, R., Provenzano, R. (2008): Hemostasis factors and aging. Exp. Gerontol. 43: 66-73.

[10]. Meade, T.W., North, W.R.S., Chakrabarti, R., Itaines, A.P., Stirling, Y. (1977): Population-based distributions of haemostatic variables. Br. Med. Bull. 33: 283-288.

[11]. Merlo Pich, V., Schmaier, A., James, G.E. (1996). Population study of platelet and aging. Br. Med. Bull. 22: 180-85

[12]. Multer, F., Mutch, N.J., Schenk, W.A. (2009). Platelet polyphosphates are pro inflammatory and procoagulant mediatiors invivo cell. 139 (6): 1143-1156.

[13]. Sapripanti and Carpi (1999). Exp Gevontol. 33 (7-8): 891-6.

[14]. Segal, J.B., Molitermo, A.R. (2006): Platelet count differ by ethinnicity sex, and age in united states, Ann Epidemiol. 16 (2) 123-30 Springer, T.A. (2011): Biology and physics of von willebrand factor concatameter. Journal of thrombosis and Heamostasis. 9 (7): $130-143$. 